\title{
The Induction and Compensation of Asymmetric Eye Movements Following Unilateral Blockage of a Horizontal Semicircular Canal in the Rabbit
}

\author{
N. H. Barmack and V. E. Pettorossi ${ }^{\mathrm{a}}$ \\ Department of Ophthalmology, Neurological Sciences Institute, Good Samaritan Hospital and Medical Center, Portland, \\ Oregon 97209
}

The influence of unilateral plugs of the left horizontal semicircular canal (LHC plugs) of rabbits on the development and compensation of asymmetric eye movements evoked by horizontal vestibular stimulation was studied. LHC plugs caused an immediate reduction of $50-65 \%$ in the gain of the horizontal vestibuloocular reflex (HVOR). This reduction in gain was achieved without altering the symmetry of the HVOR, and was accompanied by a change in the axial alignment of eye movements evoked by vestibular stimulation about the vertical (HVOR) and longitudinal (VVOR) axes. Postoperative asymmetry of eye movements developed 12-48 hr after the plugging operation. The development of asymmetry was reduced if the rabbit was restrained for $24 \mathrm{hr}$, thereby minimizing vestibular stimulation following the plugging operation. Over a 3-4 week period, the normal symmetry of eye movements was restored and the axial alignments of the HVOR and VVOR returned to the preoperative values. The gain of the HVOR did not recover. The horizontal cervicoocular reflex (HCOR) was examined before the plugging operation and after compensation of asymmetry was complete. The gain and phase of the HCOR were not altered. A relatively simple set of explanations at a cellular level is proposed to account for the induction and compensation of asymmetric eye movements following a unilateral plug of the horizontal semicircular canal.

For over a century, the oculomotor system has been used to characterize the changes in CNS function that occur following unilateral damage to the peripheral vestibular apparatus. Such unilateral damage causes both an eye nystagmus, with the slow phase towards the damaged side, as well as other postural disturbances (Bechterew, 1883; Dow, 1938; Fisch, 1973; Schaefer and Meyer, 1973; Baarsma and Collewijn, 1975; Lacour et al., 1976; Jensen, 1979). Over a period of days to weeks, these oculomotor and postural abnormalities are attenuated. It is this process of "compensation" that has stimulated neurophysiological inquiry into the effects of unilateral labyrinthectomies on oculomotor function. Compensation has served as a model for

\footnotetext{
Keceived June 15, 1987; revised Oct. 17, 1987; accepted Dec. 3, 1987.

This research was supported by Grant EY 404167 and by a grant from the Oregon Lions Sight and Hearing Foundation.

Correspondence should be addressed to Neal H. Barmack, Ph.D., Neurological Sciences Institute, 1120 N.W. 20th Avenue, Portland, OR 97209.

aresent address: Istituto di Fisiologia Umana, Universita di Perugia, Via del Giochetto, Perugia, Italy.

Copyright (C) 1988 Society for Neuroscience $0270-6474 / 88 / 082836-08 \$ 02.00 / 0$
}

understanding the more general phenomenon of plasticity of the CNS.

There are at least 2 major consequences of unilateral peripheral damage to the vestibular apparatus: (1) loss of vestibular primary afferent activity causes a reduction of synaptic drive to secondary vestibular neurons ipsilateral to the damaged labyrinth, with a consequent loss in their spontaneous activity (Precht et al., 1966; Shimazu and Precht, 1966; Maioli et al., 1983), and (2) loss of stimulus-modulated activity to both the damaged side, by vestibular primary afferents, and the intact side, through a commissural pathway, reduces the range of stimulus-driven activity of secondary vestibular neurons, particularly on the side of peripheral vestibular damage, and lowers the gain of vestibuloocular reflexes (Baarsma and Collewijn, 1975). The time course of the attenuation of nystagmus during "compensation" varies over a large range, depending on the species (Dow, 1938; Schaefer and Meyer, 1973; Maioli et al., 1983). Although the "compensation" of nystagmus (the recovery of symmetric eye movements) is complete in all species examined, the recovery of vestibuloocular reflex gains is less than complete (Baarsma and Collewijn, 1975; Wolfe and Kos, 1977; Wolfe et al., 1978; Istl et al., 1983; Maioli et al., 1983).

The technique of making a unilateral labyrinthectomy potentially confounds the study of "compensation" by causing both a loss of spontaneous vestibular primary afferent activity as well as a loss of stimulus-modulated activity. Alternatively, we have used a modification of the technique of semicircular canal plugging (Money and Scott, 1962) to block stimulus-modulated activity of the plugged canal, while leaving spontaneous primary afferent activity unaffected (Barmack, 1988). Presumably, if the primary afferent activity originating from the ampulla of the plugged semicircular canal were not reduced by the plugging operation, then no nystagmus would be caused by the operation. We have observed in the rabbit that a unilateral horizontal semicircular canal plug does not induce nystagmus, but that natural vestibular stimulation in conjunction with a unilateral plug does. A behavioral description of the induction and compensation of asymmetry constitutes the principal contribution of this report.

\section{Materials and Methods}

Preparatory surgical procedures. Twenty-one rabbits, weighing 1.0-2.0 $\mathrm{kg}$, were the subjects in this experiment. In preparatory operations the rabbits were fitted with an acrylic head-restraint device that mated with a steel rod, as previously described (Barmack, 1988).

Plugging operation of the left horizontal semicircular canal. Rabbits were anesthetized with halothane or ketamine hydrochloride $(50 \mathrm{mg} /$ 


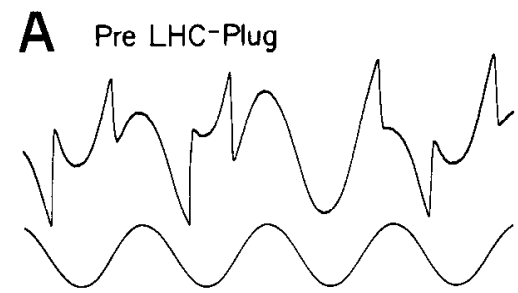

B 2.5 hours Post LHC-Plug

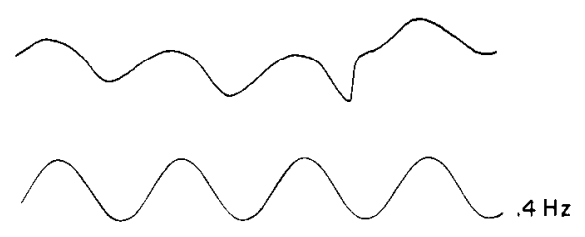

C 24 hours Post LHC-Plug<smiles>CCC1CCC1CCCCCCCCCCC1CC1CC</smiles>

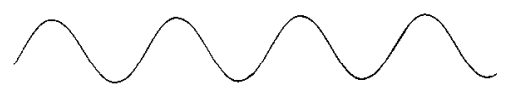



Figure 1. Influence of a plug of the left horizontal semicircular canal (LHC) on the HVOR evoked at a stimulus frequency of $0.40 \mathrm{~Hz}$ at different times following the plugging operation. $A$, Control. $B$, Immediately after the plugging operation $(2.5 \mathrm{hr})$, the gain of the HVOR was reduced, but the HVOR velocity bias was small $\left(V_{13}=0.05\right)$. $C$. At $24 \mathrm{hr}$, the HVOR velocity bias was large $\left(V_{13}=0.85\right)$, and $(D)$ at $330 \mathrm{hr}$, the HVOR velocity bias was reversed, but small $\left(V_{\mathrm{B}}=-0.08\right)$. $\mathrm{kg})$, xylazine $(6 \mathrm{mg} / \mathrm{kg})$, and acepromazine maleate $(1.2 \mathrm{mg} / \mathrm{kg})$. The technique for unilaterally plugging the HSC with small silver spindles was identical to that previously described (Barmack, 1988). The rabbits were maintained for 1 week with daily intramuscular injections of penicillin $\mathrm{G}(50,000 \mathrm{U} / \mathrm{kg})$ and prednisolone $(6 \mathrm{mg} / \mathrm{kg})$.

Eye position recording. Eye position was measured with an infrared light projection technique (Barmack, 1988). This technique has a sensitivity of $0.2^{\prime}$ of arc and is linear to within $5 \%$ for eye deviations of $\pm 15^{\circ}$. The transducer could be radially aligned to establish the optimal plane of eye movement during the horizontal vestibuloocular reflex (HVOR) or vertical vestibuloocular reflex (VVOR).

Vestibular and neck proprioceptive stimulation. Rabbits were positioned in a triaxial, servocontrolled rate table with the head fixed at the center of rotation. The body of the rabbit was encased in foam rubber and supported with elastic straps to a plastic tube aligned with the longitudinal axis. The position of the tube could be controlled independently of head position. Neck proprioceptive stimulation was provided by rotating the body independently of the head, in the horizontal plane, with the center of rotation passing through the junction of the $\mathrm{C}_{1}$ and $\mathrm{C}_{2}$ cervical vertebrae. The rate table was oscillated sinusoidally at a constant amplitude of $\pm 10^{\circ}$ over a frequency range of $0.01-0.80$ $\mathrm{Hz}$ about the earth vertical axis (HVOR), earth horizontal axis (VVOR), or by rotating the body in the horizontal plane relative to the fixed head (HCOR). Table position signals were obtained from servopotentiometers for each axis of rotation. During vestibular and neck proprioceptive stimulation, the vision of both eyes of the rabbit was always completely occluded by 3 layers of black cloth.

Head restraint of rabbits to prevent horizontal vestibular stimulation. In order to determine the relative importance of horizontal vestibular stimulation in the induction of asymmetric eye movements, it was necessary to prevent vestibular stimulation over a period of $24 \mathrm{hr}$. Horizontal head restraint was accomplished by maintaining the rabbits in a plastic rabbit restrainer (Nalgene) with an adjustable neck support and a curved support for the spine. The head of the rabbit was held rigid in the horizontal plane by a piece of spring steel attached to the rabbit restrainer and mated with the surgically implanted head bolts. The spring steel was flexible enough to bend in the vertical plane, but stiff enough, if unopposed by the rabbit, to maintain the head in an orientation that placed the plane of the horizontal semicircular canals in an earth-horizontal orientation.

Vestibuloocular and cervicoocular reflex testing. The gains $(G)$ of the HVOR and VVOR, as well as of the horizontal cervicoocular reflex (HCOR), were determined from measurements of the peak eye velocities attained during each half-cycle of sinusoidal rotation, as described previously (Barmack, 1988). The phases of the HVOR, VVOR, and HCOR were measured using table and eye position signals for each half-cycle of rotation. At stimulation frequencies below $0.02 \mathrm{~Hz}, 2$ cycles of stimulation were usually measured. At frequencies above $0.04 \mathrm{~Hz}, 3$ or more cycles were usually measured.

\section{Results}

HVOR measured 12-40 hr after plugging the $L H C$.

The HVOR was measured in rabbits before and at various intervals after a plug was placed in the left horizontal semicircular canal (LHC). Rather than use spontaneous nystagmus as a measure of relative imbalance of the vestibular system, we employed a more sensitive measure of the gain of the HVOR in both the left and right directions during sinusoidal oscillation of the rate table about the vertical axis (yaw). In a normal rabbits, the gain of the HVOR in both directions was symmetrical (Figs. 1A, 2). However, if the HVOR was measured $12-40 \mathrm{hr}$ after plugging the LHC, then not only was a spontaneous nystagmus of 2-15 $\mathrm{deg} / \mathrm{sec}$ present, but the HVOR became asymmetric (Гigs. $1 C$, 2). In Figure 2 the HVOR was measured in 4 "plugged" rabbits before and $12-40 \mathrm{hr}$ after the plugs were inserted. These rabbits had a spontaneous nystagmus, with a mean velocity of $3 \mathrm{deg} /$ sec. This nystagmus made measurement and interpretation of eye movements evoked at low-stimulus frequencies $(0.01-0.02$ $\mathrm{Hz}$ ) uncertain, since the stimulus-evoked eye velocities at these frequencies were low relative to the spontaneous velocity of the nystagmus. The velocity of eye movements evoked during vestibular stimulation of the plugged rabbits was "corrected" for spontaneous nystagmus by subtracting it from the peak velocities of eye movements evoked in both the left and right directions (filled symbols connected by dashed lines, Fig. 2). The LHC plugging operation caused a reduction in $G_{\mathrm{L}}$ and $G_{\mathrm{R}}$ of $50-65 \%$, an increase in phase lead of $10^{\circ}-25^{\circ}$ for leftward eye movements, and a smaller increase for rightward eye movements. In sum, these rabbits evinced the classic signs characteristic of hemilabyrinthectomized animals.

\section{HVOR measured 1-10 hr after plugging the LHC}

In contrast to a previous report of spontaneous nystagmus in squirrel monkeys evoked immediately following a unilateral plugging operation performed with the bone dust-filling technique (Paige, 1983), rabbits with unilateral silver spindle plugs evinced no spontaneous nystagmus, nor did they have an asymmetric HVOR during the first $10 \mathrm{hr}$ after the plugging operation. The gains of the HVOR, $G_{\mathrm{L}}$ and $G_{\mathrm{R}}$, were reduced by $50-65 \%$ 

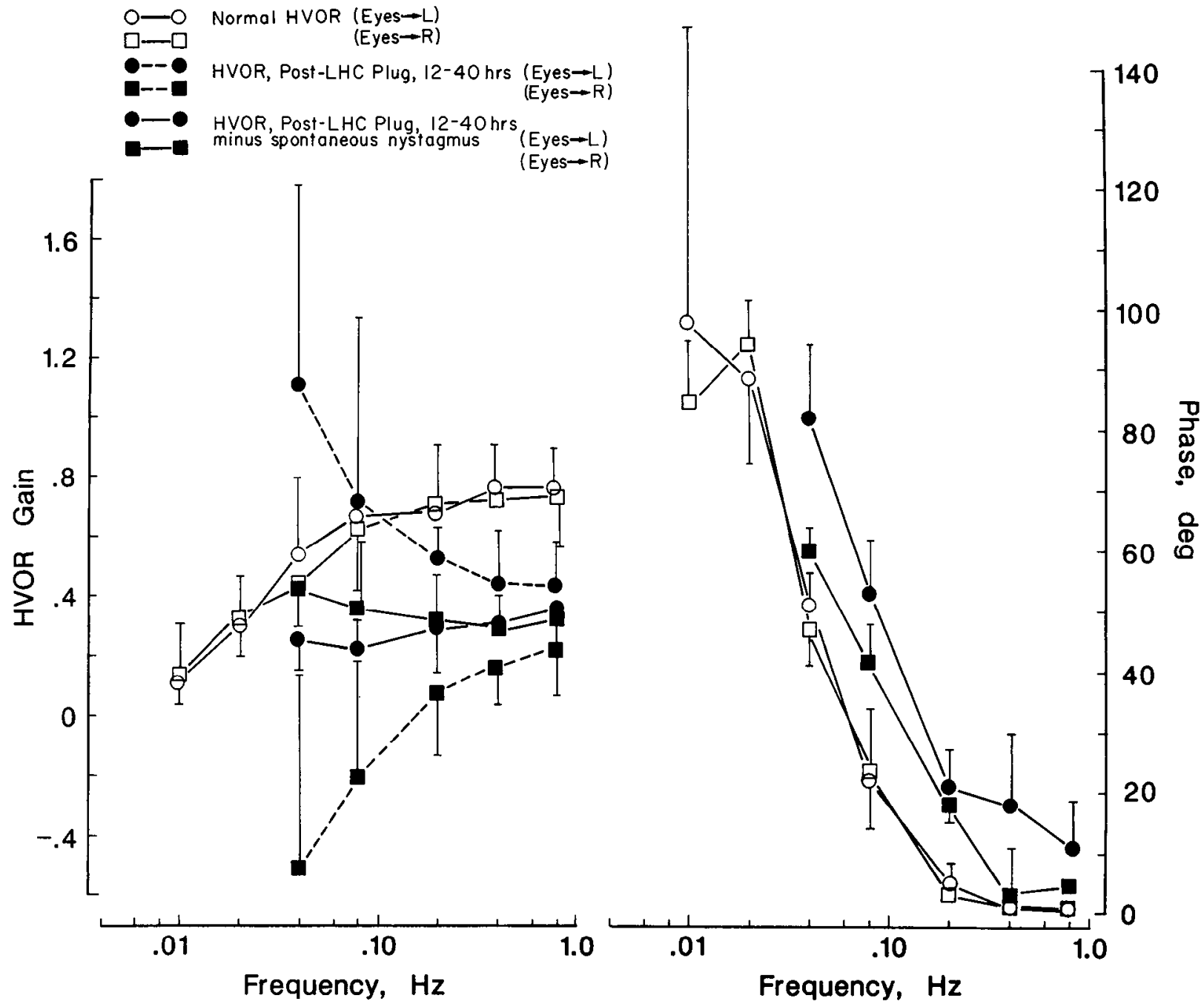

Figure 2. Influence of an LHC plug on the gain and phase of the HVOR. The HVOR was measured $12-40 \mathrm{hr}$ after a plugging operation in 4 rabbits. Each rabbit evinced a spontaneous nystagmus (slow phase to the left) during this period. The gain and phase were measured for each halfcycle of sinusoidal stimulation ( $\pm 10^{\circ}$ amplitude). Open symbols connected by solid lines, the gain and phase of the HVOR. Filled symbols connected by dashed lines, the gain and phase that were uncorrected for spontaneous nystagmus. Filled symbols connected by solid lines, corrected responses. One standard deviation is illustrated for each data point, unless the data point encompasses the SD.

depending on stimulus frequency, and this reduction in gain was accompanied by an increase in phase lead of $10^{\circ}-20^{\circ}$ (Fig. 3). After $10 \mathrm{hr}$, a nystagmus developed. It became fully manifested after $24 \mathrm{hr}$ (Figs. 1, 2). Three weeks after the LHC was plugged, the asymmetry had a slight bias in the oppositc dircction (to the right). However, there was no statistically significant increase (2-tailed Student's $t$ test) in the gains of the HVOR (Fig. 3).

\section{Changes in the axial alignment of eye movement caused by} plugging the $\mathrm{LHC}$

The axial alignments of eye movements evoked by vestibular stimulation about the vertical axis (HVOR) and about the longitudinal axis (VVOR) were measured both before and after the LHC was plugged. When tested within $10 \mathrm{hr}$ following the plugging operation, and before there was any marked asymmetry of evoked eye movements, the axial alignment of vestibularly evoked eye movements was shifted. When the right eye moved to the right, it had a downward component, and when it moved to the left, it had an upward component (Fig. 4). Similarly, there was a change in the alignment of the vertical axis of eye move- ments evoked by vestibular stimulation about the longitudinal axis. When the right eye moved down, it moved to the right, and when it moved up, it moved to the left. The axial alignment of the principal axes of horizontal and vertical eye movements reached $68^{\circ} 24-48 \mathrm{hr}$ following the LHC plugging opcration. This compares with a preoperative axial alignment for horizontally and vertically evoked eye movements of approximately $86^{\circ}$ Over a period of $30 \mathrm{~d}$, the axial alignment of evoked eye movements reverted to the preoperative value (Fig. 4).

Comparison between post-LHC plug forced vestibular stimulation and the absence of vestibular stimulation in the induction of nystagmus

The induction of asymmetry in the HVOR appeared to occur over a period of 10-24 hr, presumably as a consequence of asymmetric vestibular stimulation experienced by the rabbits with LHC plugs when they moved their heads. We examined directly the role of this asymmetric vestibular stimulation in the induction of vestibular asymmetry by restraining head movements of 4 rabbits with plugs of the LHC (see Materials and 



Figure 3. Comparison between HVOR evoked immediately after an LHC plug with the HVOR evoked at least 3 weeks after the plugging operation. The HVOR was measured in 5 rabbits within $10 \mathrm{hr}$ following the plugging operation (filled symbols). During this period there was no asymmetry of the HVOR. The rabbits were retested 3-5 weeks after the plugging operation (open symbols connected by dashed lines). During this period, there was no recovery of the gain of the HVOR. One standard deviation is illustrated for each data point.

Methods) over a $24 \mathrm{hr}$ period and comparing the time course of the induction of asymmetry in these rabbits with that of a group of 4 rabbits that received $8 \mathrm{hr}$ of continuous sinusoidal, vestibular stimulation $\left(0.4 \mathrm{~Hz} \pm 10^{\circ}\right)$ immediately following the LHC plugging operation. Although both groups of rabbits developed an asymmetry of evoked eye movements that reached a maximum $24 \mathrm{hr}$ after the plugging operation, the rabbits that received $8 \mathrm{hr}$ of continuous vestibular stimulation developed a larger asymmetry. The rabbits that were deprived of horizontal vestibular stimulation in between testing sessions developed a smaller asymmetry than did the rabbits that received $8 \mathrm{hr}$ of continuous vestibular stimulation (Fig. 5). However, the "deprivation" of vestibular stimulation was not complete because even the "deprived" rabbits received 20 min of vestibular stimulation during each vestibular testing session.

\section{Examination of a possible role for neck proprioceptors in the compensation of asymmetry}

The question of how the asymmetry of eye movements induced by the asymmetrical vestibular stimulation caused by unilateral LHC plugs is compensated for was examined with the specific intent of determining whether neck proprioceptors might "substitute" for the absence of a modulated primary vestibular afferent input from the left labyrinth. If such a substitution oc- curred, then the HCOR could potentially develop an asymmetry that could complement the vestibular asymmetry. The HCOR was tested in a group of 6 rabbits before the LHC plugging operation and 3-5 weeks after the operation, when the asymmetry of eye movements caused by the plugging operation had disappeared. The gain of the HCOR in both directions was unmodified by the plugging operation, although the gain of the HVOR was reduced to $0.4-0.5$ of the preoperative value (Figs. $6,7)$. These data indicate that neck proprioceptors could, in principle, provide information concerning asymmetry of head movements, but that the gain of the HCOR is not used to compensate for the asymmetry in gain of the HVOR.

\section{Discussion}

Vestibular contributions to the induction of asymmetrical eye movements

One of our main observations is that a unilateral plug of the horizontal semicircular canal without the concomitant experience of vestibular stimulation does not induce asymmetric eye movements over the range of stimulus frequencies and amplitudes used in the present experiment. Although the plugging operation caused an immediate frequency-dependent reduction in $G_{\mathrm{L}}$ and $G_{\mathrm{R}}$ (Fig. 3), the induction of asymmetry occurred only after rabbits with LHC plugs received horizontal vestibular 


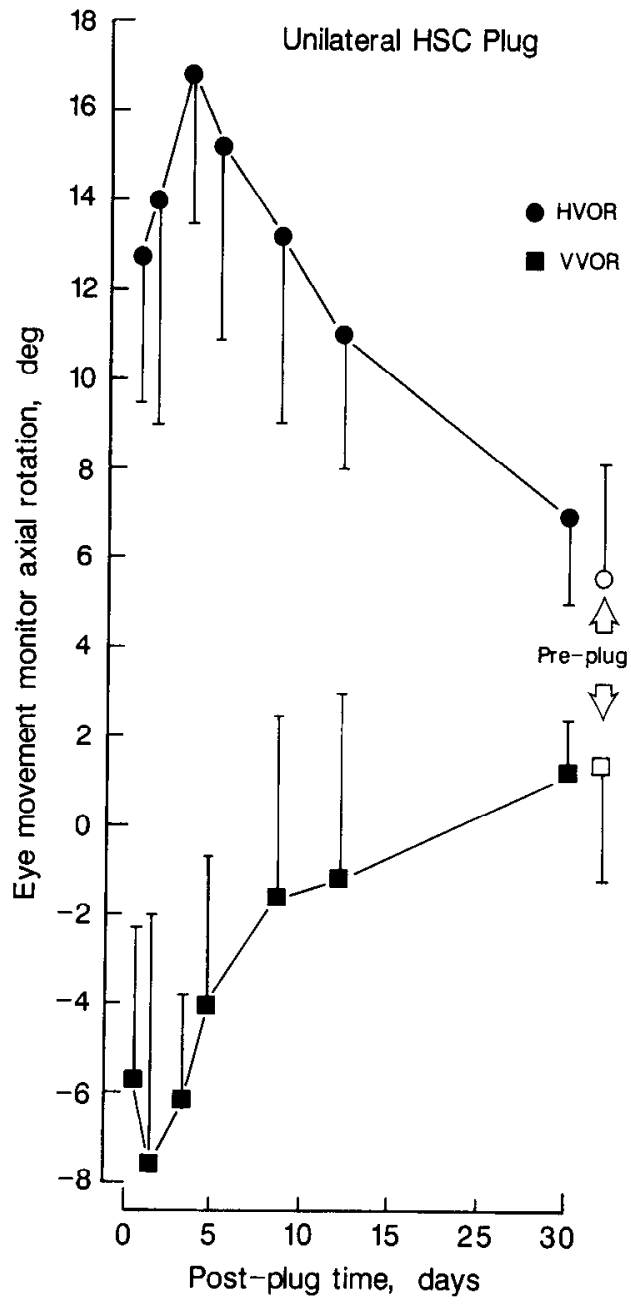

Figure 4. Changes in the axes of eye movements caused by a plug of the LHC. The alignment axes for the HVOR (circles) and VVOR (squares) were measured at stimulus frequencies of $0.4 \mathrm{~Hz}$ in 5 rabbits before (open symbols) and at different times after the plugs were inserted. When the HVOR was measured $24 \mathrm{hr}$ after the plug was inserted, the right eye moved down when it moved to the right. During the VVOR, the right eye moved to the right when it moved down. The recovery of normal axial alignment was virtually complete within 4 weeks. One standard deviation is illustrated for each data point.

stimulation. This induction of asymmetry of eye movements developed over 10-24 $\mathrm{hr}$ and then gradually subsided over a period of $10-25 \mathrm{~d}$. These data differ from the observations made concerning the immediate effects of unilateral horizontal semicircular canal plugs in monkeys (Paige, 1983). In monkeys a nystagmus with the slow phase directed toward the plugged canal was immediately observed following the plugging operation. The techniques used in performing the plugging operations in the present experiment and in the experiments on monkeys were dissimilar. In the present experiment, the LHC was plugged with a small silver spindle that gently compressed the membranous horizontal semicircular canal. This technique produces reversible inactivation of the LHC (Barmack, 1988). In the monkey, the bone dust generated by the burr of a dental drill was mixed with bone wax and used to compress the membranous horizontal semicircular canal (Paige, 1983). This procedure may have been more traumatic than the procedure of using silver wire spindles, since it subjected the cristae ampullares to rela-

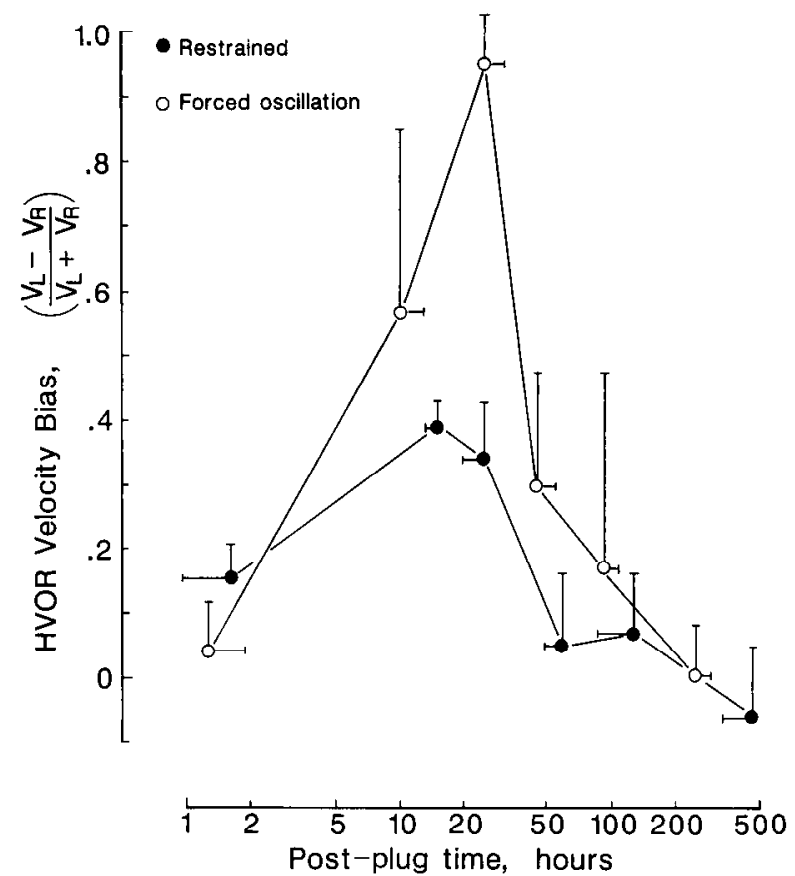

Figure 5. Influence of vestibular stimulation on the development of nystagmus after an LHC plug. Following the plugging operation, 4 rabbits were immediately placed in the rate table and subjected to $8 \mathrm{hr}$ of horizontal sinusoidal vestibular stimulation at $0.40 \mathrm{~Hz} \pm 10^{\circ}$ ("forced oscillation," unfilled circles). At different times following the operation, the gain of the HVOR was measured at a stimulus frequency of 0.40 $\mathrm{Hz}$. At the conclusion of the forced oscillation and in between subsequent tests, the rabbits were maintained in conventional cages in which they could freely move. Following the plugging operation, 4 rabbits were restrained in a "rabbit restrainer," which prevented horizontal vestibular stimulation. The gain of the HVOR at $0.40 \mathrm{~Hz}$ was also measured in these rabbits, and after testing they were returned to the rabbit restrainer for the first $24 \mathrm{hr}$ postoperative period. Subsequently they were returned to rabbit cages in between tests, which allowed free mobility. Standard deviations are illustrated for each data point.

tively large and repeated pressure transients while the canal was first drilled open and then compressed with bone dust. In the present experiment, the postoperative use of steroids might have reduced both temporary and permanent damage to the labyrinth caused by the plugging operation.

In the squirrel monkey experiments, in spite of the use of a potentially more traumatic plugging procedure, the spontaneous nystagmus that was present immediately after the plugging operation nevertheless increased in velocity over the first 24-48 hr postoperatively. Furthermore, the bias of the HVOR was greater at higher stimulus frequencies than would be predicted by the linear addition of the velocity of the spontaneous nystagmus. It was concluded that the response asymmetry was caused by a static nonlinearity and an amplitude-dependent DC component (Paige, 1983). In the present experiment, the maximum-velocity spontaneous nystagmus was determined for each rabbit. This was subtracted from the evoked eye velocity at each stimulus frequency (Fig. 2). This spontaneous velocity appeared to account for the velocity bias of the HVOR when it was measured $12-40 \mathrm{hr}$ after the plugging operation, even at higher stimulus frequencies. The apparent difference between this finding and the work on squirrel monkeys, in which it was necessary to invoke an amplitude-dependent DC component, can probably be attributed to the procedures and criteria used to measure the velocity of spontaneous nystagmus. 

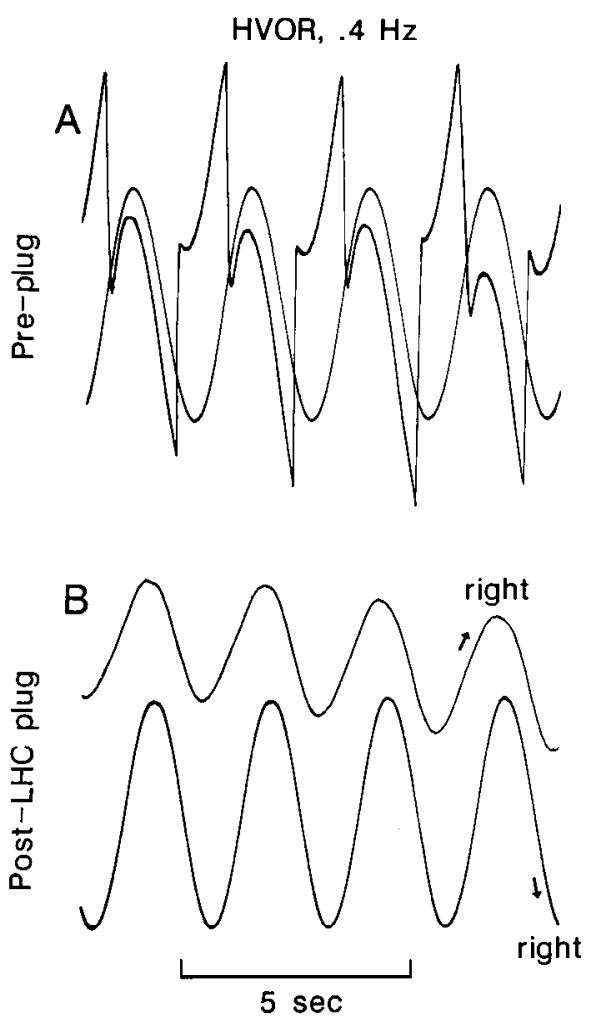

HCOR, .04 Hz

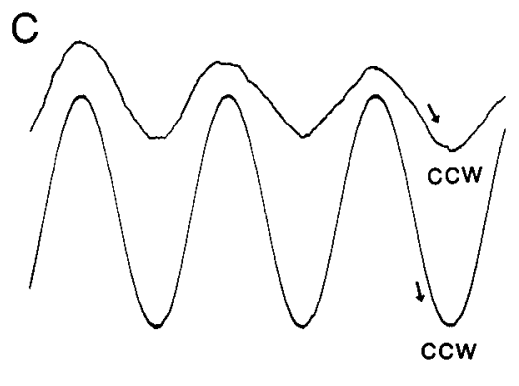

D

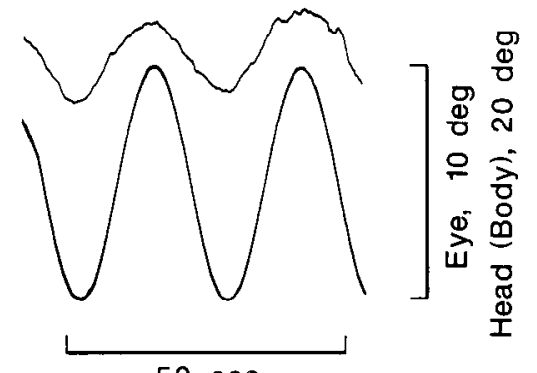

$50 \mathrm{sec}$
Figure 6. Comparison of the HVOR and HCOR before and after a plug of the LHC. The HVOR and HCOR are illustrated before and 3 weeks after the LHC was plugged. The gain of the HVOR was reduced, but the gain of the HCOR remained normal in both directions.
In rabbits, the initial absence of spontaneous postoperative nystagmus, its subsequent induction by vestibular stimulation, and its delayed induction under conditions of reduced vestibular stimulation indicate that the nystagmus is a consequence of a stimulus-dependent modification of the HVOR, rather than a direct consequence of the plugging operation. This contrasts with the findings on squirrel monkeys, in which an immediate postoperative nystagmus was caused by the plugging operation (Paige, 1983).

\section{$L H C$ plug and reduction of the gain of the $H V O R$}

Although the induction of asymmetric eye movements following the unilateral plugging operation was dependent on vestibular stimulation, the reduction in gain of the HVOR was immediate and permanent (50-65\%). Similarly, unilateral plugs of the horizontal semicircular canal of squirrel monkeys caused an immediate gain reduction of 46-60\% (cf. Fig. 3; Paige, 1983). However, in monkeys, after the first postoperative week, there was a significant, although incomplete, recovery in the gain of the HVOR. In rabbits, after a postoperative recovery period of more than 3 weeks, there was no statistically significant recovery of the gain of the HVOR (Fig. 3). This finding is consistent with a previous observation that unilaterally labyrinthectomized rabbits evinced no sign of recovery of the gain of the HVOR when tested 6 months postoperatively (Baarsma and Collewijn, 1975). In spite of the lack of recovery of gain, there was complete compensation of the asymmetry of the HVOR and complete recovery of the normal axial alignment of the HVOR and VVOR within a 3-4 week postoperative period.

In human patients with absent labyrinthine function, there is some evidence that neck proprioceptive signals may be enhanced to compensate for absent vestibular inputs during head rotation (Kasai and Zee, 1978). In the present experiment, we could find no evidence that neck proprioceptive inputs influ- enced the recovery of the HVOR from the induced asymmetry. The HCOR values in LHC-plugged rabbits were equal in phase and gain to preoperative values, and these were similar to previous measurements of gain and phase of the HCOR (Barmack et al., 1981). It is possible, although, we think, unlikely, that neck proprioceptive signals contribute to the recovery in gain of the HVOR, but that the present experiment did not extend the postoperative recovery period long enough to observe this recovery.

\section{Mechanisms of induction and compensation of HVOR asymmetry}

The induction of asymmetric eye movements in rabbits with unilateral LHC plugs depended on vestibular stimulation. This observation indicates that the site of induction must be centrally located. The induction of asymmetry cannot be ascribed to a traumatically induced imbalance in primary afferent activity. What kinds of neuronal interactions could account for this altered vestibuloocular function? The lack of stimulus-dependent modulation of hair cell responses or of primary vestibular afferent activity may be sufficient by itself to reduce the amount of transmitter released spontaneously, to decrease the number of postsynaptic receptors, or to decrease the sensitivity of postsynaptic receptors. The hypothetical influence of synaptic activity on the total number or aggregation of postsynaptic receptors is illustrated in Figure $8 \mathrm{~A}$.

Autoradiographic and biochemical evidence suggests that glutamate or aspartate may be synaptic transmitters in the cat vestibular nerve (Raymond et al., 1984). Electrical stimulation of a glutaminergic pathway in the hippocampus causes an increase in glutamate postsynaptic receptors (Baudry et al., 1980). However, at present it is not known what parameters of neuronal activity are critical for producing this effect.

Alternatively, it is possible that a change in the excitability 
Figure 7. Influence of LHC plugs on the gain and phase of the HCOR. The HCOR was measured in 6 rabbits before (circles) and 3-5 weeks after (squares) the LHC was plugged. There was no significant alteration of the HCOR as a consequence of the unilateral plugging operation. One standard deviation is illustrated for the upper and lower data points at each stimulus frequency.

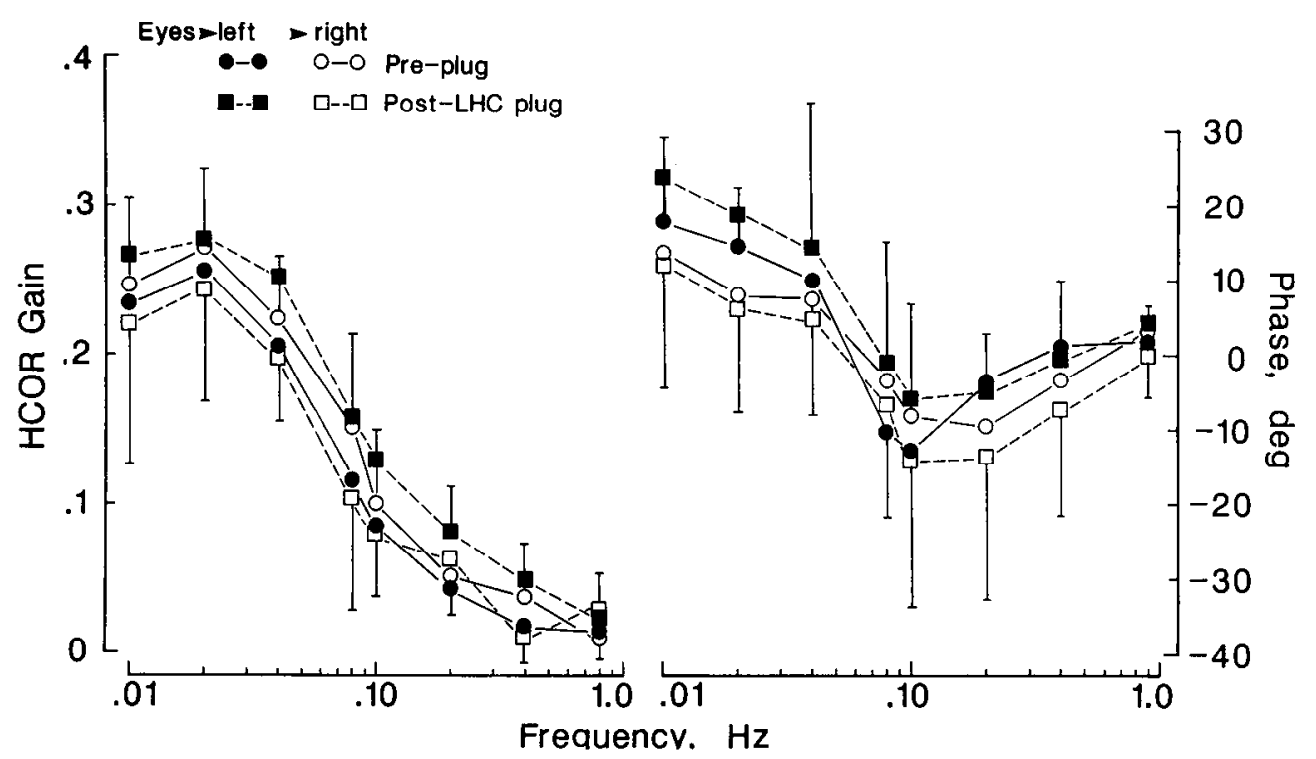

of secondary vestibular neurons could be induced through synaptic competition (Fig. $8 B$ ). Such a mechanism has been proposed to account for the biased development of ocular dominance columns in the visual cortex of cats and monkeys that are deprived of binocular vision (Wiesel and Hubel, 1963; Guillery and Stelzner, 1970). The physiological mechanism that could actually account for a more active set of synapses occupying the postsynaptic area formerly occupied by another set of less active synapses is unknown. In Figure $8 B$, this hypothetical mecha- nism is illustrated as a displacement of postsynaptic receptors that receive an unvarying excitatory synaptic input by postsynaptic receptors that receive a varying inhibitory synaptic input. In this instance, the presynaptic inhibitory neuron could correspond to a commissural neuron originating in the vestibular nucleus, which is contralateral to the LHC plug. The synaptic competition would cause this commissural input to become more potent in regulating the activity of the postysynaptic neuron. Although there is compelling evidence that the vestibular
Figure 8. Possible mechanisms of induction of and compensation for asymmetric eye movements. $A$, Lack of stimulus modulation of primary afferent activity causes a diffusion or loss of postsynaptic receptors, rendering the postsynaptic neuron less sensitive to a maintained spontaneous level of presynaptic activity. $B$, Synaptic "competition" between 2 different presynaptic inputs (excitatory input, open symbol; inhibitory input, filled symbol) renders the unmodulated input less competitive in maintaining postsynaptic receptor density. $C$, Recurrent inhibitory feedback of postsynaptic neuron "compensates" for an induced lower output activity by withdrawing inhibition due to decreased activity from the output neuron.
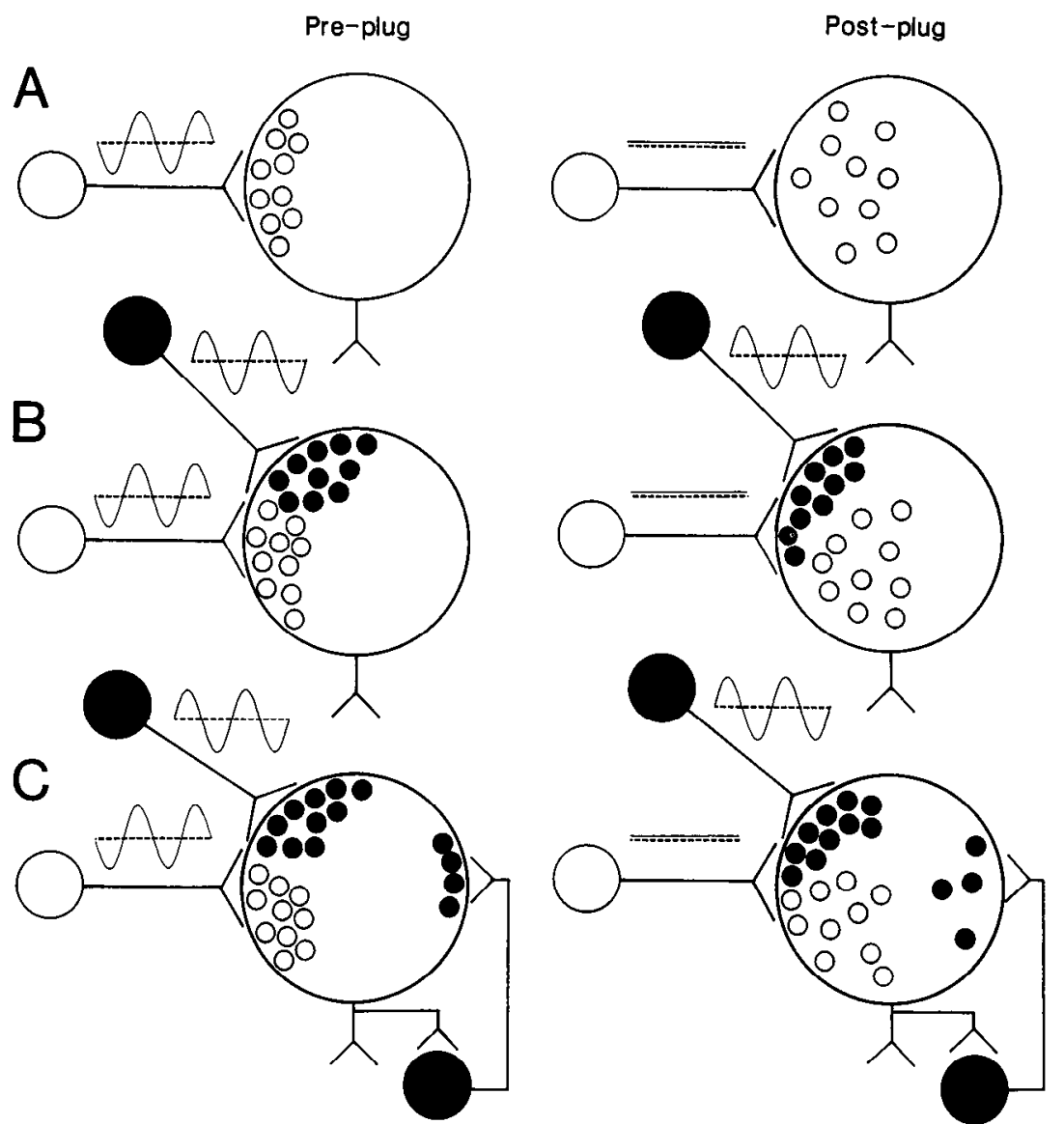
commissural system may play a role in the compensation of asymmetry (Dieringer and Precht, 1977; Bienhold and Flohr, 1978; Galiana et al., 1984), at present there is no direct evidence that would indicate whether postsynaptic receptors of secondary vestibular neurons are lost or displaced as a consequence of a unilateral semicircular canal-plugging operation. Nor is there direct evidence that the phenomenon of induction of asymmetry of eye movements can be attributed purely to either the action of commissural neurons or the action of these neurons on postsynaptic receptors.

Synaptic inactivity or competition could account for the induction of asymmetry of eye movements. However, once induced, what factors contribute to compensation or restoration of symmetry? If a recurrent inhibitory neuron were coupled to the output of a secondary vestibular neuron, then, as the output of the secondary vestibular neuron decreased, the effective recurrent inhibition would also decrease (Fig. 8C). Conversely, an excitatory vestibular commissural pathway (not illustrated) might increase in efficacy in driving secondary vestibular neurons that are ipsilateral to a unilaterally sectioned vestibular nerve (or plugged semicircular canal), as has been demonstrated in the frog (Dicringer and Precht, 1977). Consequently, the output of a secondary neuron could eventually be stabilized about some constant frequency. The time necessary for this to occur might correspond to a period of compensation. The mechanism discussed here depends on the growth or aggregation of postsynaptic receptors under certain conditions of presynaptic activity; for the purpose of emphasis, we ignore other possible mechanisms, such as changes in presynaptic release, changes in presynaptic innervation density (Korte and Friedrich, 1979), or induced dendritic growth. While, in the past, these mechanisms have not been differentiated electrophysiologically, it is possible that, with the advent of more selective immunocytochemical markers, the problem of identifying mechanisms of plasticity may not be so intractable in the future.

\section{References}

Baarsma, E. $\Lambda$., and H. Collewijn (1975) Changes in compensatory eye movements after unilateral labyrinthectomy in the rabbit. Arch. Otorhinolaryngol. 211: 219-230.

Barmack, N. H. (1988) The influence of temporary blockage of semicircular canals on the loss and recovery of vestibuloocular reflexes. J. Neurosci. 8: 2816-2826.

Barmack, N. H., M. A. Nastos, and V. E. Pettorossi (1981) The horizontal and vertical cervico-ocular reflexes of the rabbit. Brain Res. 224: 261-278.

Baudry, M., M. Oliver, R. Creager, A. Wieraszko, and G. Lynch (1980) Increase in glutamate receptors following repetitive electrical stimulation in hippocampal slices. I.ife Sci. 27: 325-330.

Bechterew, W. von (1883) Ergebnisse der Durchschneidung des $N$. acusticus, nebst Erörterung der Bedeutung der semicirculären Kanäle für das Körpergleichgewicht. Pfluegers Arch. 30: 312-347.

Bienhold, H., and H. Flohr (1978) Role of commissural connexions between vestibular nuclei in compensation following unilateral labyrinthectomy. J. Physiol. (Lond.) 284: 178P.

Dieringer, N., and W. Precht (1977) Modification of synaptic input following unilateral labyrinthectomy. Nature 269: 431-433.

Dow, R. S. (1938) The effects of unilateral and bilateràl labyrinthectomy in monkey, baboon and chimpanzee. Am. J. Physiol. 121:392399.

Fisch, U. (1973) The vestibular response following unilateral vestibular neurectomy. Acta Otolaryngol. (Stockh.) 76: 229-238.

Galiana, H. L., II. Flohr, and G. Melvill Jones (1984) A reevaluation of intervestibular nuclear coupling: Its role in vestibular compensation. J. Neurophysiol. 51: 242-259.

Guillery, R. W., and D. J. Stelzner (1970) The differential effects of unilateral lid closure upon the monocular and binocular segments of the dorsal lateral geniculate nucleus in the cat. J. Comp. Neur. 139: 412-422.

Istl, Y. E., D. Hyden, and D. W. F. Schwarz (1983) Quantification and localization of vestibular loss in unilaterally labyrinthectomized patients using a precise rotatory test. Acta Otolaryngol. (Stockh.) 96: 437-445.

Jensen, D. W. (1979) Reflex control of acute postural asymmetry and compensatory symmetry after a unilateral vestibular lesion. Neuroscience 4: 1059-1073.

Kasai, T., and D. S. Zee (1978) Eye-head coordination in labyrinthincdefective human beings. Brain Res. 144: 123-141.

Korte, G. E., and V. L. Friedrich (1979) The fine structure of the feline superior vestibular nucleus: Identification and synaptology of the primary vestibular afferents. Brain Res. 176: 3-32.

Lacour, M., J. P. Roll, and M. Appaix (1976) Modifications and development of spinal reflexes in the alert baboon (Papio papio) following an unilateral vestibular neurotomy. Brain Res. 113: 255-269.

Maioli, C., W. Precht, and S. Ried (1983) Short- and long-term modifications of vestibulo-ocular response dynamics following unilateral vestibular nerve lesions in the cat. Exp. Brain Res. 50: 259-274.

Money, K. E., and J. W. Scott (1962) Functions of separate sensory receptors of nonauditory labyrinth of the cat. Am. J. Physiol. 202: $1211-1220$

Paige, G. D. (1983) Vestibuloocular reflex and its interactions with visual following mechanisms in the squirrel monkey. II. Response characteristics and plasticity following unilateral inactivation of horizontal canal. J. Neurophysiol. 49: 152-168.

Precht, W., H. Shimazu, and C. H. Markham (1966) A mechanism of central compensation of vestibular function following hemilabyrinthectomy. J. Neurophysiol. 29: 996-1010.

Raymond, J., A. Nieoullon, D. Dememes, and A. Sans (1984) Evidence for glutamate as a neurotransmitter in the cat vestibular nerve: Radioautographic and biochemical studies. Exp. Brain Res. 56: $523-$ 531.

Schaefer, K. P., and D. L. Meyer (1973) Compensatory mechanisms following labyrinthine lesions in the guinea-pig. A simple model of learning. In Memory and Transfer of Information, H. P. Zippel, ed., pp. 203-232, Plenum, New York.

Shimazu, H., and W. Precht (1966) Inhibition of central vestibular neurons from the contralateral labyrinth and its mediating pathway. J. Neurophysiol. 29: 467-492.

Wiesel, T., and D. Hubel (1963) Single-cell responses in striate cortex of kittens deprived of vision in one eye. J. Neurophysiol. 26: 10021017.

Wolfe, J. W., and C. M. Kos (1977) Nystagmic responses of the rhesus monkey to rotational stimulation following unilateral labyrinthectomy: Final report. Trans. Am. Acad. Ophthalmol. Otolaryngol. 84: $38-45$.

Wolfe, J. W., E. J. Engelken, and C. N. Kos (1978) Low-frequency harmonic acceleration as a test of labyrinthine function: Basic methods and illustrative cases. Trans. Am. Acad. Ophthalmol. Otolaryngol. 86: 130-142. 